\title{
Conversion of carboxylic acids to amides under the action of $\operatorname{tantalum}(\mathrm{V})$ chloride
}

\author{
Azat M. Gabdullin,* Oleg S. Mozgovoj, Rita N. Kadikova, Ilfir R. Ramazanov, Aliya K. Amirova and \\ Usein M. Dzhemilev
}

Institute of Petrochemistry and Catalysis of Russian Academy of Sciences, 141 Prospekt Oktyabrya, 450075 Ufa, Russia; skill15@mail.ru (O.S.M.); kadikritan@gmail.com (R.N.K); ilfir.ramazanov@gmail.com (I.R.R.); alya02265@gmail.com (A.K.A.); ink@anrb.ru (U.M.D.)

* Correspondence: saogabdullinsao@gmail.com

\section{Introduction}

It is known that tantalum compounds can be useful for the synthesis of various classes of organic compounds. The $\mathrm{TaCl}_{5}-\mathrm{Mg}$ reagent system is an effective tool for the reduction of nonfunctionalized alkynes and 1-alkynyl sulfones to the corresponding olefins [1]. Recently, we have developed a regio- and stereoselective method for the synthesis of substituted 3-alkenyl amines and 4-alkynylols based on the reduction of alkynyl amines and alcohols using a similar reagent system $\mathrm{NbCl}_{5}-\mathrm{Mg}$ [2]. The possibility of creating a new carbon-carbon bond was demonstrated by the example of the $\mathrm{TaCl}_{5}$-catalyzed carbomagnesiation reaction of 1-alkenes with $n$-alkyl Grignard reagents [3-5]. Thus, low-valence tantalum complexes are effective reagents for the transformation of the triple bond of various acetylenic compounds. The closest electronic analogue of the tantalum atom is niobium. According to [6], $\mathrm{NbCl}_{5}$ promotes the conversion of carboxylic acids into carboxamides. In this work, in order to study the possibility of conversion of carboxylic acids under the conditions of organotantalum synthesis, we studied the reaction of carboxylic acids with secondary amines in the presence of catalytic amounts of $\mathrm{TaCl}_{5}$.

\section{Results and discussion}

We found that the reaction of carboxylic acids 1 with 3 equivalents of a secondary amine in the presence of $33 \mathrm{~mol}$. \% of $\mathrm{TaCl}_{5}$ in a solution of methylene chloride after refluxing for 5 hours gave amides 2 in 51-87\% yield (Table 1). In the case of amination of heptanoic acid with dibenzylamine, the yield of carboxamide was $51 \%$ (Entry 4). Apparently, the decrease in the yield of the formed amide in the case of dibenzylamine is associated with steric hindrances arising from the interaction of heptanoyl chloride formed in situ with dibenzylamine. At present, we have failed to obtain betulinic acid amide in methylene chloride solution. It is possible that the inertness of betulinic acid is caused by the presence of hydroxyl group in the A ring that binds $\mathrm{TaCl}_{5}$ reagent. The quantitative formation of oxoniobium and oxotitanium carboxylates as a result of the treatment of carboxylic acids with $\mathrm{NbCl}_{5}$ and $\mathrm{TiCl}_{4}$ is described in the literature $[7,8]$. However, the generation of oxotitanium carboxylates requires the use of more amount of carboxylic acid than the formation of oxoniobium carboxylates. At the same time, the effect of steric factors on the yield of the formed amide (31\%) was demonstrated by the example of the $\mathrm{TiCl}_{4}$-promoted amination reaction of pivalic acid using pyrrolidine in a tetrahydrofuran solution [9]. The reaction of pivalic acid with diethylamine in a solution of methylene chloride in the presence of $\mathrm{NbCl}_{5}$ gave the corresponding amide in $78 \%$ yield [6]. 
Table $1 \mathrm{MHz}$ and ${ }^{13} \mathrm{C}-\{1 \mathrm{H}\} \quad \mathrm{NMR}$ spectra at $100 \mathrm{MHz}$ in $\mathrm{CDCl}_{3}$. The chemical shifts are reported in ppm relative to tetramethylsilane (TMS) as the internal standard. The numbering of atoms in the ${ }^{13} \mathrm{C}-\{1 \mathrm{H}\}$ and

$33 \mathrm{~mol} . \% \mathrm{TaCl}_{5}$ $\mathrm{R}_{2}{ }_{2} \mathrm{NH}$ (3 equiv.)

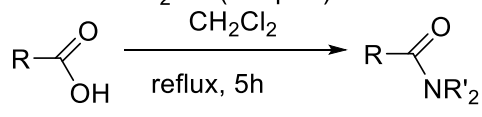

1

\begin{tabular}{cccc}
\hline Entry & $\mathrm{R}-K_{\mathrm{OH}, \mathbf{1}}^{\mathrm{O}}$ & $\mathrm{R}_{2}{ }_{2} \mathrm{NH}$ & \multicolumn{1}{l}{$\begin{array}{l}\text { Isolated yeld } \\
\mathbf{2 ,} \%\end{array}$} \\
\hline 1 & $\mathrm{R}=n$ Pent & $\mathrm{R}=n \mathrm{Bu}$ & $\mathbf{2 a}(77)$ \\
2 & $\mathrm{R}=n \mathrm{Hept}$ & $\mathrm{R}=n \mathrm{Bu}$ & $\mathbf{2 b}(81)$ \\
3 & $\mathrm{R}=n \mathrm{Hex}$ & $\mathrm{R}=n \mathrm{Bu}$ & $\mathbf{2 c}(83)$ \\
4 & $\mathrm{R}=n \mathrm{Hex}$ & $\mathrm{R}=\mathrm{Bn}$ & $\mathbf{2 d}(51)$ \\
5 & $\mathrm{R}=\mathrm{C}_{15} \mathrm{H}_{31}$ & $\mathrm{R}=n \mathrm{Bu}$ & $\mathbf{2 e}(86)$ \\
6 & $\mathrm{R}=\mathrm{C}_{17} \mathrm{H}_{35}$ & $\mathrm{R}=n \mathrm{Bu}$ & $\mathbf{2 f}(87)$
\end{tabular}

$\mathrm{TaCl}_{5}$-promoted reaction of succinic acid $\mathbf{3}$ with diethylamine in methylene chloride solution gave dicarboxamide 4 in 53\% yield (Scheme 1). In the present work, we also found that the reaction under study allows the selective conversion of 1adamantanecarboxylic acid under the action of diethylamine to the corresponding amide 5 .

$$
\begin{aligned}
& \overbrace{\mathrm{OH}}^{\mathrm{O}} \\
& \begin{array}{c}
\underset{33 \mathrm{~mol} . \% \mathrm{TaCl}_{5}}{\mathrm{Et}_{2} \mathrm{NH}(3 \text { equiv. })} \\
\underset{\text { reflux, } 6 \mathrm{~h}}{\mathrm{CH}_{2} \mathrm{Cl}_{2}}
\end{array}
\end{aligned}
$$

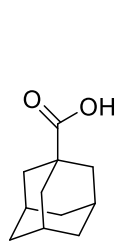

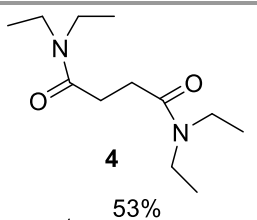
$53 \%$

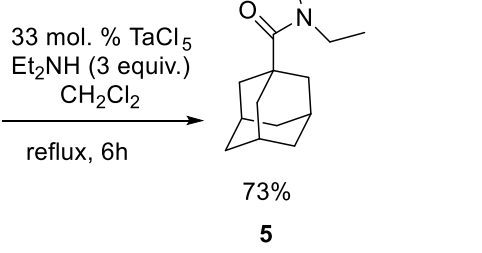

Scheme 1 Conversion of carboxylic acids to amides under the action of tantalum(V) chloride.

\section{Conclusions}

Thus, we have demonstrated for the first time that the reaction of aliphatic mono- and dicarboxylic acids with secondary amines under the action of catalytic amounts of tantalum (V) chloride leads to the selective formation of carboxamides.

\section{Experimental section}

\section{General information}

The carboxylic acids and secondary amines were obtained from Sigma-Aldrich or Acros. Dichloromethane were distilled over $\mathrm{P}_{2} \mathrm{O}_{5}$. Nuclear magnetic resonance spectroscopy was performed on a Brucker Avance 500. The ${ }^{1} \mathrm{H}$ NMR spectra were recorded at 500
${ }^{1} \mathrm{H}$ NMR spectra of the compounds $\mathbf{2 a - f ,} \mathbf{4}, \mathbf{5}$ is shown in Figures 1. Elemental analysis was performed using a Carlo-Erba CHN 1106 elemental analyser. Mass spectra were obtained on a Finnigan 4021 instrument. The yields were calculated from the isolated amount of carboxamides obtained from starting 2-alkynylamines.

Preparation of carboxamides $\mathbf{2 a - f ,} \mathbf{4 , 5}$ via conversion of carboxylic acids to amides under the action of tantalum(V) chloride.

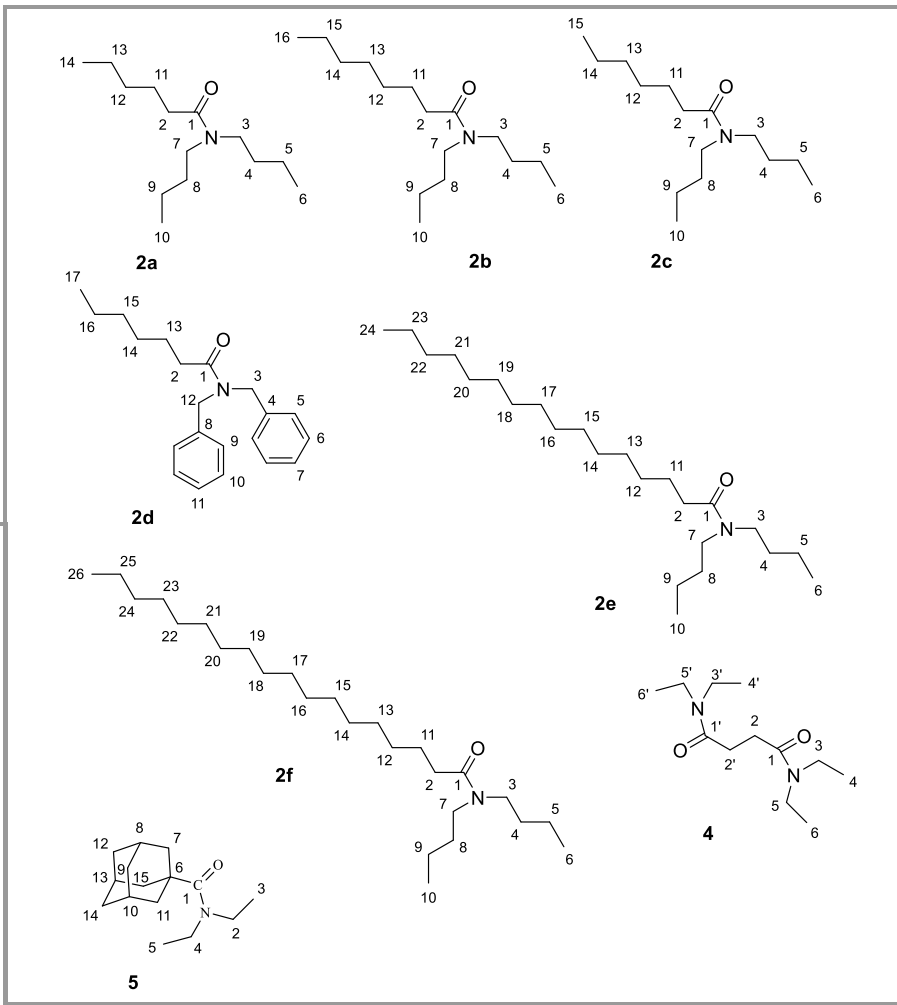

Figure 1 The numbering of atoms in the ${ }^{13} \mathrm{C}$ - and ${ }^{1} \mathrm{H}-\mathrm{NMR}$ spectra of the compounds $2 a-f, \mathbf{4}, \mathbf{5}$.

$N, N$-dibutylhexanamide; Typical Procedure.

In a $50.0 \mathrm{~mL}$ round-bottomed flask equipped with a magnetic stirrer, argon-inlet, and a reflux condenser $\mathrm{TaCl}_{5}(238 \mathrm{mg}, 0.666$ mmol) was added followed by addition of a soln in $\mathrm{CH}_{2} \mathrm{Cl}_{2}(4.0 \mathrm{~mL})$, of the hexanoic acid $(232 \mathrm{mg}, 2.0 \mathrm{mmol})$. After a few $\mathrm{min}$ of vigorous stirring, a suspension formed and dibutylamine, $(258 \mathrm{mg}$, $5.26 \mathrm{mmol}$ ) was introduced into the reaction mixture. In $0.5 \mathrm{~h}$, the temperature was slowly raised to $45^{\circ} \mathrm{C}$ and the reaction time was maintained $5 \mathrm{~h}$ or $6 \mathrm{~h}$ at $45{ }^{\circ} \mathrm{C}$. The reaction mixture was diluted with $\mathrm{Et}_{2} \mathrm{O}(5 \mathrm{~mL})$, and $25 \mathrm{wt} \% \mathrm{KOH}$ solution $(3 \mathrm{~mL})$ was added dropwise while the reaction flask was cooled in an ice bath. The aqueous layer was extracted with diethyl ether $(3 \times 10 \mathrm{~mL})$. The combined organic layers were washed with brine $(20 \mathrm{~mL})$, dried over anhydrous $\mathrm{MgSO}_{4}$. The residue was distilled through a micro column at $20 \mathrm{mmHg}$ to give $2 \mathrm{a}$ ( $248 \mathrm{mg}, 77 \%$ ) as a colourless oil. b.p. $77-79{ }^{\circ} \mathrm{C}(20 \mathrm{mmHg})$. Evaporation of solvent and purification of the residue by column chromatography (hexane/ethyl acetate, $5: 1$ ) gave a yellow oil; yield: $350 \mathrm{mg},(77 \%) ; R_{f}=0.69$ (hexane/ethyl acetate, 5:1). 
${ }^{1} \mathrm{H}$ NMR $\left(500 \mathrm{MHz}, \mathrm{CDCl}_{3}\right): \delta=0.89-0.98(\mathrm{~m}, 9 \mathrm{H}), 1.28-1.36(\mathrm{~m}$, $6 \mathrm{H}), 1.47-1.57(\mathrm{~m}, 4 \mathrm{H}), 1.62-1.68(\mathrm{~m}, 2 \mathrm{H}), 2.29(\mathrm{t}, J=7.6 \mathrm{~Hz}, 2 \mathrm{H})$, $3.22(\mathrm{t}, J=7.6 \mathrm{~Hz}, 2 \mathrm{H}), 3.31(\mathrm{t}, J=7.7 \mathrm{~Hz}, 2 \mathrm{H})$.

${ }^{13} \mathrm{C} \mathrm{NMR}\left(500 \mathrm{MHz}, \mathrm{CDCl}_{3}\right): \delta=13.83,13.89,13.98,20.11,20.27$, $22.54,25.23,29.95,31.29,31.72,33.13,45.61,47.73,172.68$.

MS (EI): m/z, \% = 227 (3) [M+], 184 (6), 128 (17), 100 (10), 86 (100), 43 (17).

Anal. calcd for $\mathrm{C}_{14} \mathrm{H}_{29} \mathrm{NO},(\%)$ : $\mathrm{C}, 73.95 ; \mathrm{H}, 12.86 ; \mathrm{N}, 6.16$. Found, \%: C, 74.11; H, 13.01; N, 5.99.

\section{$\mathrm{N}, \mathrm{N}$-dibutyloctanamide (2b)}

Using the procedure described above $288 \mathrm{mg}$ of octanoic acid (2 mmol) gave crude product that was purified by column chromatography (hexane : ethyl acetate $=5: 1$ ) to afford $\mathbf{2 b}(413$ $\mathrm{mg}, 81 \%)$ as colorless oil. $\mathrm{R}_{\mathrm{f}} 0.67$.

MS (EI): m/z, \% = 255 (5) $\left[\mathrm{M}^{+}\right], 226$ (2), 184 (11), 128 (37), 100 (15), 86 (100), 41 (18).

Anal. calcd for $\mathrm{C}_{16} \mathrm{H}_{33} \mathrm{NO}$, (\%): C, 75.23; $\mathrm{H}, 13.02 ; \mathrm{N}, 5.48$; Found, \%: C, $75.31 ; \mathrm{H}, 13.11 ; \mathrm{N}, 5.33$.

\section{$\mathrm{N}, \mathrm{N}$-dibutylheptanamide $(2 \mathrm{c})$}

Using the procedure described above $260 \mathrm{mg}$ of heptanoic acid (2 $\mathrm{mmol}$ ) gave crude product that was purified by column chromatography (hexane : ethyl acetate $=5: 1)$ to afford 2 c $(400$ $\mathrm{mg}, 83 \%$ ) as colorless oil. $\mathrm{R}_{\mathrm{f}} 0.70$.

${ }^{1} \mathrm{H}$ NMR $\left(500 \mathrm{MHz}, \mathrm{CDCl}_{3}\right): \delta=0.88-0.98(\mathrm{~m}, 9 \mathrm{H}), 1.27-1.36(\mathrm{~m}$, $8 \mathrm{H}), 1.47-1.56(\mathrm{~m}, 4 \mathrm{H}), 1.61-1.68(\mathrm{~m}, 2 \mathrm{H}), 2.29(\mathrm{t}, J=7.4 \mathrm{~Hz}, 2 \mathrm{H})$, $3.22(\mathrm{t}, J=7.7 \mathrm{~Hz}, 2 \mathrm{H}), 3.31(\mathrm{t}, J=7.6 \mathrm{~Hz}, 2 \mathrm{H})$.

${ }^{13} \mathrm{C} \mathrm{NMR}\left(500 \mathrm{MHz}, \mathrm{CDCl}_{3}\right): \delta=13.83,13.89,14.05,20.11,20.27$, $22.54,25.52,29.20,29.94,31.29,31.70,33.18,45.61,47.74$, 172.72.

MS (EI): m/z, \% = 241 (4) [M+ $], 198$ (4), 184 (11), 128 (34), 100 (12), $86(100), 43(26)$.

Anal. calcd for $\mathrm{C}_{15} \mathrm{H}_{31} \mathrm{NO}$, (\%): C, 74.63; $\mathrm{H}, 12.94 ; \mathrm{N}, 5.80$; Found, \%: C, $74.71 ; \mathrm{H}, 12.87 ; \mathrm{N}, 6.01$.

\section{$\mathrm{N}, \mathrm{N}$-dibenzylheptanamide (2d)}

Using the procedure described above $260 \mathrm{mg}$ of heptanoic acid (2 $\mathrm{mmol}$ ) and dibenzylamine (394 $\mathrm{mg}, 2 \mathrm{mmol}$ ) gave crude product that was purified by column chromatography (hexane : ethyl acetate $=5: 1)$ to afford $\mathbf{2 d}(315 \mathrm{mg}, 51 \%)$ as colorless oil. $R_{f} 0.57$.

${ }^{1} \mathrm{H} \mathrm{NMR}\left(500 \mathrm{MHz}, \mathrm{CDCl}_{3}\right): \delta=0.89(\mathrm{t}, J=6.8 \mathrm{~Hz}, 3 \mathrm{H}), 1.27-1.31(\mathrm{~m}$, $4 \mathrm{H}), 1.33-1.38(\mathrm{~m}, 2 \mathrm{H}), 1.71-1.77(\mathrm{~m}, 2 \mathrm{H}), 2.44(\mathrm{t}, J=7.4 \mathrm{~Hz}, 2 \mathrm{H})$, $4.47(\mathrm{~s}, 2 \mathrm{H}), 4.63(\mathrm{~s}, 2 \mathrm{H}), 7.18(\mathrm{~d}, J=7.5 \mathrm{~Hz}, 2 \mathrm{H}), 7.24(\mathrm{~d}, J=7.4 \mathrm{~Hz}$, $2 \mathrm{H}), 7.28-7.30(\mathrm{~m}, 2 \mathrm{H}), 7.32-7.35(\mathrm{~m}, 2 \mathrm{H}), 7.39(\mathrm{t}, J=7.4 \mathrm{~Hz}, 2 \mathrm{H})$.

${ }^{13} \mathrm{C} \mathrm{NMR}\left(500 \mathrm{MHz}, \mathrm{CDCl}_{3}\right): \delta=14.05,22.54,25.44,29.12,31.63$, $33.29,48.05,49.92,126.39$ (2C), 128.29 (2C), 127.34, 127.58, $128.58(2 \mathrm{C}), 128.94(2 \mathrm{C})$.

MS (EI): m/z, \% = 309 (4) [M+], 218 (38), $148(3), 106$ (100), 43 (18).

Anal. calcd for $\mathrm{C}_{21} \mathrm{H}_{27} \mathrm{NO}$, (\%): C, 81.51; $\mathrm{H}, 8.79 ; \mathrm{N}, 4.53$; Found, \%: $\mathrm{C}$, $81.61 ; \mathrm{H}, 8.57 ; \mathrm{N}, 4.51$

\section{$N, N$-dibutylpalmitamide (2e)}

Using the procedure described above $512 \mathrm{mg}$ of palmitic acid (2 $\mathrm{mmol}$ ) and dibutylamine, $(258 \mathrm{mg}, 5.26 \mathrm{mmol}$ ) gave crude product that was purified by column chromatography (hexane : ethyl acetate $=5: 1)$ to afford $2 \mathbf{e}(633 \mathrm{mg}, 86 \%)$ as colorless oil. $R_{f} 0.66$.

${ }^{1} \mathrm{H} \mathrm{NMR}\left(500 \mathrm{MHz}, \mathrm{CDCl}_{3}\right): \delta=0.87-0.97(\mathrm{~m}, 9 \mathrm{H}), 1.26(\mathrm{~s}, 18 \mathrm{H}), 1.29-$ $1.35(\mathrm{~m}, 10 \mathrm{H}), 1.47-1.57(\mathrm{~m}, 4 \mathrm{H}), 1.61-1.65(\mathrm{~m}, 2 \mathrm{H}), 2.28(\mathrm{t}, J=$ $7.5 \mathrm{~Hz}, 2 \mathrm{H}), 3.21(\mathrm{t}, J=7.6 \mathrm{~Hz}, 2 \mathrm{H}), 3.31(\mathrm{t}, J=7.5 \mathrm{~Hz}, 2 \mathrm{H})$.

${ }^{13} \mathrm{C} \mathrm{NMR}\left(500 \mathrm{MHz}, \mathrm{CDCl}_{3}\right): \delta=13.83,13.89,14.10,20.11,20.27$, $22.68,25.54,29.36,29.49,29.53$ (2C), 29.65 (3C), 29.68 (3C), 29.95, $31.29,31.92,33.17,45.59,47.73,172.67$.

MS (EI): m/z, \% = 368 (2) [M+ $], 240$ (2), 184 (17), 156 (62), 128 (94), 86 (100), 43 (31).

Anal. calcd for $\mathrm{C}_{24} \mathrm{H}_{49} \mathrm{NO}$, (\%): C, 78.40; $\mathrm{H}, 13.43 ; \mathrm{N}, 3.81$; Found, \%: C, 78.52; H, 13.45; N, 3.63.

\section{$\mathrm{N}, \mathrm{N}$-dibutylstearamide (2f)}

Using the procedure described above $568 \mathrm{mg}$ of stearic acid (2 $\mathrm{mmol}$ ) and dibutylamine, $(258 \mathrm{mg}, 5.26 \mathrm{mmol})$ gave crude product that was purified by column chromatography (hexane : ethyl acetate $=5: 1)$ to afford $\mathbf{2 f}(689 \mathrm{mg}, 87 \%)$ as colorless oil. $R_{f} 0.69$.

${ }^{1} \mathrm{H}$ NMR $\left(500 \mathrm{MHz}, \mathrm{CDCl}_{3}\right): \delta=0.86-0.96(\mathrm{~m}, 9 \mathrm{H}), 1.25(\mathrm{~s}, 22 \mathrm{H}), 1.28$ $-1.33(\mathrm{~m}, 10 \mathrm{H}), 1.47-1.54(\mathrm{~m}, 4 \mathrm{H}), 1.59-1.64(\mathrm{~m}, 2 \mathrm{H}), 2.26(\mathrm{t}, J=$ $7.3 \mathrm{~Hz}, 2 \mathrm{H}), 3.19(\mathrm{t}, J=7.3 \mathrm{~Hz}, 2 \mathrm{H}), 3.29(\mathrm{t}, J=7.2 \mathrm{~Hz}, 2 \mathrm{H})$.

${ }^{13} \mathrm{C} \mathrm{NMR}\left(500 \mathrm{MHz}, \mathrm{CDCl}_{3}\right): \delta=13.81,13.86,14.09,20.09,20.25$, $22.67,25.51,29.35,29.49,29.52$ (2C), 29.64 (3C), 29.68 (5C), 29.93, $31.28,31.91,33.14,45.57,47.70,172.62$.

Anal. calcd for $\mathrm{C}_{26} \mathrm{H}_{53} \mathrm{NO}$ (\%): C, 78.92; $\mathrm{H}, 13.50 ; \mathrm{N}, 3.54$; Found, \%: C, 79.07; H, 13.39; N, 3.61.

\section{$N^{1}, N^{1}, N^{4}, N^{4}$-tetraethylsuccinamide (4)}

Using the procedure described above $236 \mathrm{mg}$ of succinic acid (2 $\mathrm{mmol}$ ) and diethylamine, (146 mg, $5.26 \mathrm{mmol}$ ) gave crude product that was purified by column chromatography (hexane : ethyl acetate $=5: 1)$ to afford $4(242 \mathrm{mg}, 53 \%)$ as colorless oil. $R_{f} 0.69$.

${ }^{1} \mathrm{H}$ NMR $\left(500 \mathrm{MHz}, \mathrm{CDCl}_{3}\right): \delta=1.53(\mathrm{t}, J=7.1 \mathrm{~Hz}, 6 \mathrm{H}), 1.22(\mathrm{t}, J=7.1$ $\mathrm{Hz}, 6 \mathrm{H}), 2.69(\mathrm{~s}, 4 \mathrm{H}), 3.37-3.41(\mathrm{q}, J=7.1 \mathrm{~Hz}, \mathrm{~J}=7.2 \mathrm{~Hz}, 6 \mathrm{H})$.

${ }^{13} \mathrm{C} \mathrm{NMR}\left(500 \mathrm{MHz}, \mathrm{CDCl}_{3}\right): \delta=13.12(2 \mathrm{C}), 14.23(2 \mathrm{C}), 28.26(2 \mathrm{C})$, $40.30(2 \mathrm{C}), 41.90(2 \mathrm{C}), 171.26(2 \mathrm{C})$.

Anal. calcd for $\mathrm{C}_{12} \mathrm{H}_{24} \mathrm{~N}_{2} \mathrm{O}_{2}$, (\%): C, 63.12; $\mathrm{H}, 10.59 ; \mathrm{N}, 12.27$; Found, \%: C, 63.21; H, 11.13; N, 12.18 .

\section{$(3 r, 5 r, 7 r)-N, N$-diethyladamantane-1-carboxamide (5)}

Using the procedure described above $360 \mathrm{mg}$ of $(3 r, 5 r, 7 r)$ adamantane-1-carboxylic acid ( $2 \mathrm{mmol}$ ) and diethylamine, $(146 \mathrm{mg}$, $5.26 \mathrm{mmol}$ ) gave crude product that was purified by column chromatography (hexane : ethyl acetate $=5: 1$ ) to afford $5(343 \mathrm{mg}$, $73 \%)$ as colorless oil. $R_{f} 0.64$.

${ }^{1} \mathrm{H} \mathrm{NMR}\left(500 \mathrm{MHz}, \mathrm{CDCl}_{3}\right): \delta=1.53(\mathrm{t}, J=6.8 \mathrm{~Hz}, 6 \mathrm{H}), 1.74$ (br. $\left.\mathrm{s}, 6 \mathrm{H}\right)$, 2.01 (br.s, 6H), 2.05 (br.s, 6H), 2.05 (br.s, 3H), 3.44 (br.s, 4H). 
${ }^{13} \mathrm{C} \mathrm{NMR}\left(500 \mathrm{MHz}, \mathrm{CDCl}_{3}\right): \delta=13.72(2 \mathrm{C}), 28.62(3 \mathrm{C}), 36.72(3 \mathrm{C})$, 38.68, $39.18(3 \mathrm{C}), 41.81$ (2C), 176.09 .

MS (EI): m/z, \% = 235 (22) [M+], 206 (5), 135 (100), 93 (12), 79 (14), $41(6)$.

Anal. calcd for $\mathrm{C}_{15} \mathrm{H}_{25} \mathrm{NO}$, (\%): $\mathrm{C}, 76.55 ; \mathrm{H}, 10.71 ; \mathrm{N}, 5.95$; Found, \%: C, 76.61; H, 10.68; N, 4.89 .

\section{Conflicts of interest}

The authors declare no competing financial interest.

Funding: This work was financially supported by the Russian Science Foundation (grant No. 19-73-10113).

\section{Acknowledgements}

The structural studies of all compounds were performed with the use of Collective Usage Centre "Agidel" of Ufa Research of Russian Academy of Science at the Institute Petrochemistry and Catalysis (AAAA-A19-119022290004-8).

\section{References}

1 Kataoka, Y. Generation and Synthetic Applications of Niobium- and Tantalum-Alkyne Complexes 1992.

2 Kadikova, R. N.; Gabdullin, A. M.; Mozgovoj, O. S.; Ramazanov, I. R.; Dzhemilev, U. M. $\mathrm{NbCl}_{5}-\mathrm{Mg}$ Reagent System in Regio- and Stereoselective Synthesis of (2Z)Alkenylamines and (3Z)-Alkenylols from Substituted 2Alkynylamines and 3-Alkynylols. Molecules 2021, 26, 3722.

3 Sultanov, R. M.; Ismagilov, R. R.; Popod'ko, N. R.; Tulyabaev, A. R.; Dzhemilev, U. M. $\mathrm{TaCl}_{5}$-catalyzed reaction of 1-alkenes with $n$-alkyl Grignard reagents. J. Organomet. Chem. 2013, 724, 51-56.

4 Sultanov, R. M.; Ismagilov, R. R.; Popod'ko, N. R.; Tulyabaev, A. R.; Sabirov, D.; Dzhemilev, U. M. $\mathrm{TaCl}_{5}$-catalyzed carbomagnesiation of some norbornenes with ethyl Grignard reagents. J. Organomet. Chem. 2013, 745-746:120.

5 Sultanov, R. M.; Dzhemilev, U. M.; Samoilova, E. V.; Ismagilov, R. R.; Khalilov, L. M.; Popod'ko, N. R. Two routes of tantalum-catalyzed alkene carbomagnesiation with ethyl Grignard reagents. Journal of Organometallic Chemistry. 2012, 715, 5 - 8 .

6 Nery, M. S.; Ribeiro, R. P.; Lopes, C. C.; Lopes, R. S. C. Niobium Pentachloride Promoted Conversion of Carboxylic Acids to Carboxamides: Synthesis of the 4-Aryl-1,2,3,4tetrahydroisoquinoline Alkaloid Structures. Synthesis (Stuttg) 2003, 2, 272-276.

7 Brown, D. A; Wallbridge, M. G. H.; Alcock, N.W. Preparation of Some Oxoniobium Carboxylates. X-Ray Crystal and Molecular Structure of $[\{\mathrm{NbCl},(\mathrm{O}, \mathrm{CPh})), \mathrm{O}]$. J. CHEM. soc. Dalt. TRANS 1993, 1, xxiii-xxviii.

8 Kapoor, R.; Sharma, R.; Kapoor, P. Indian J. Chem., Sect. A $1985,24,761$.

9 WILSON, J. D.; EINGARTEN, H. Titanium tetrachloride promoted conversion of carboxylic acids to earboxamides. Can. J. Chem. 1970, 48, 983-986. 